\title{
A Comparison of Benzodiazepine, Serotonin, and Dopamine Agents in the Taste-Reactivity Paradigm
}

\author{
DALLAS TREIT
}

Department of Psychology, University of Alberta, Edmonton, Alberta, Canada T6G 2E9

AND

KENT C. BERRIDGE

Department of Psychology, University of Michigan, Ann Arbor, MI 48104-1687

Received 9 May 1990

\begin{abstract}
TREIT, D. AND K. C. BERRIDGE. A comparison of benzodiazepine, serotonin, and dopamine agents in the taste-reactivity paradigm. PHARMACOL BIOCHEM BEHAV 37(3) 451-456, 1990. - Previous studies have shown that rats' positive, palatabilitydependent consummatory reactions to infused tastes are selectively facilitated by a benzodiazepine agonist (chlordiazepoxide), and that this effect can be blocked by the coadministration of benzodiazepine antagonists (e.g., Ro 15-1788). The purpose of the present study was to determine whether agents acting at other receptor sites (dopaminergic, serotonergic), which have been shown to modulate food consumption, might also modify rats' palatability-dependent reactivity to infused tastes. In this experiment, the benzodiazepine agonist, diazepam, facilitated positive palatability reactions, while dopaminergic agents (haloperidol, apomorphine, amphetamine) had no significant effects on either positive or aversive reactions. The putative 5-HT $1 \mathrm{~A}$ agonists, buspirone and gepirone, had a general inhibitory action on both positive and aversive palatability reactions. These results are surprising in view of the effects of serotonergic and dopaminergic agents on food and fluid intake. Our results suggest that the benzodiazepine receptor system may play a special role in the neural control of appetite through its enhancement of the positive palatability of tastes. Dopamine systems, by contrast, appear to control food intake by modulating processes that are independent of food affect evaluation.
\end{abstract}

Taste reactivity Palatability Diazepam Apomorphine Amphetamine Haloperidol Buspirone Gepirone

IT has been known for some time that anxiolytics such as the benzodiazepines facilitate food and fluid intake [for reviews see $(8,10,32)]$. Until recently, the functional mechanisms by which this facilitation occurs have remained unclear. One problem has been that simple intake measures cannot discriminate between two of the most probable mechanisms: 1) appetite enhancement, or 2) anxiety reduction. Whereas appetite enhancement would increase intake directly, by activating systems involved in normal hunger, anxiety reduction would increase intake indirectly, by reducing "anxiety" which would compete with, inhibit, or otherwise suppress consumption. Simple intake could be affected in a similar way by either or both of these mechanisms and therefore is inadequate to discriminate between the two mechanisms (32).

Recent research using the taste reactivity paradigm developed by Grill and Norgren (20) has suggested that benzodiazepine-type anxiolytics increase food and fluid intake by directly enhancing the positive palatability of tastes. In the taste infusion paradigm (20), solutions of different taste (e.g., sucrose, quinine) are infused into the rat's mouth through intraoral cannulae. The affective, speciestypical reactions of the rat to these infused tastes (e.g., tongue protrusions, passive drips, gapes) can be characterized as hedonic (i.e., "positive" or "ingestive"), neutral, or aversive, and correspond closely to rats' normal preference for solutions of these tastes (20). More importantly, this profile of taste reactions is particularly sensitive to variables that directly affect palatability, but is relatively insensitive to variables that control intake by altering other processes [e.g., "anxiety," $(5,6,25)]$.

In a study that applied this taste-reactivity technique to ingestive psychopharmacology, the prototypical benzodiazepine, chlordiazepoxide, selectively increased hedonic (i.e., "positive") taste reactions while having little or no effect on "neutral" or "aversive" reactions to the same tastes (4). This direct enhancement of positive palatability by chlordiazepoxide was counteracted by the benzodiazepine receptor antagonists Ro 15-1788 and CGS 8216 (33), and occurred even in chronic mesencephalic decerebrate rats (2). These data suggest that specific benzodiazepine receptor systems (21) modulate the positive palatability of tastes, and in this way directly increase "appetite."

The extent to which agents acting at other receptor systems modulate palatability also warrants investigation. There is good reason to suspect, for example, that dopaminergic and serotonergic systems could be involved in the modulation of palatability. 
Dopaminergic agonists such as apomorphine and amphetamine have potent suppressive effects on the food and fluid intake of rats at moderate to high doses [e.g., $(1,23,30)]$, and at lower doses, amphetamine can facilitate intake $(15,35)$. Disruption of dopaminergic transmission, either with neuroleptic drugs or lesions of dopamine systems, dramatically reduces food and fluid intake (17, $18,28,31,34)$. Disruption of dopamine transmission also suppresses reward-related instrumental behavior, so strongly as to have been described as producing "anhedonia," or a reduced capacity to generate positive hedonic affect in response to food or other rewards $(36,37)$. Agents which interact with serotonergic systems, such as the putative $5-\mathrm{HT}_{1 \mathrm{~A}}$ agonists buspirone and gepirone, have recently been found to facilitate the food intake of rats $(7,9,12-14,24)$.

It is not known, however, whether the effects of dopaminergic and serotonergic agents on rats' intake are due to a direct effect on palatability, or to side-effects on general activity, arousal, incentive attribution, or other processes that could affect intake. The taste infusion paradigm would allow a more direct assessment of the effects of these agents on palatability, in the same way as it was used to characterize the direct effect of chlordiazepoxide on palatability. In addition to clarifying the possible role of dopaminergic and serotonergic systems in palatability, a comparison of benzodiazepine and nonbenzodiazepine agents on palatability would further characterize the drug-class specificity of the enhancement of palatability by benzodiazepines.

METHOD

\section{Subjects}

Twenty male Sprague-Dawley rats $(250-350 \mathrm{~g})$ served as subjects. The rats were individually housed throughout the experiment with free access to food and water.

\section{Cannulae Implantation}

The rats were anaesthetized with ketamine $(100 \mathrm{mg} / \mathrm{kg})$ and xylazine $(10 \mathrm{mg} / \mathrm{kg})$ and implanted with bilateral, intraoral cannulae according to the procedures described previously $(2-4,20$, 33). Briefly, the cannulae enter the head dorsally, and are anchored to the skull with stainless steel screws and acrylic cement. They enter the mouth lateral to the first maxillary molar. To allow the infusion of taste solutions, fine tubing (PE 10) is fitted inside the cannulae.

\section{Taste Stimuli and Drug Administration}

The taste stimuli were $0.3 \mathrm{M}$ sucrose, $0.03 \mathrm{M}$ sucrose, and $3 \times 10^{-4} \mathrm{M}$ quinine hydrochloride. The two sucrose stimuli primarily elicit varying degrees of hedonic taste reactions, while the quinine stimulus primarily elicits aversive reactions.

Each rat received one taste stimulus per day. Each taste was presented on two consecutive days: first, with the low dose of a drug, second, with the high dose of the same drug. Taste order was randomized across these pairs of days. Twenty min before the taste infusion, rats were injected intraperitoneally with either saline $(0.5$ $\mathrm{ml})$, diazepam $(2.5$ or $5.0 \mathrm{mg} / \mathrm{kg})$, apomorphine $(0.5$ or 1.0 $\mathrm{mg} / \mathrm{kg})$, d-amphetamine $(0.25$ or $1.5 \mathrm{mg} / \mathrm{kg})$, haloperidol $(0.5$ or $1.0 \mathrm{mg} / \mathrm{kg}$ ), buspirone ( 1.0 or $2.0 \mathrm{mg} / \mathrm{kg}$ ), or gepirone (1.0 or 3.0 $\mathrm{mg} / \mathrm{kg}$ ). The order of drug administration was randomized, with two exceptions: 1) buspirone and gepirone were administered last and in that order, 2) the lower dose of each drug was administered first for a particular taste, followed on the next day by the higher dose. Saline control trials were repeated 4-5 times for each taste over the course of the experiment, and these control data were averaged into a single saline score for each rat. All drugs were dissolved in sterile saline, except diazepam which was dissolved in propylene glycol and ethyl alcohol. Prior to testing, each rat received three injections of $5 \mathrm{mg} / \mathrm{kg}$ of diazepam over three consecutive days in order to minimize any initial sedative effects of this drug [cf. (2)].

\section{Apparatus}

On each test day, the rat's cannulae were connected to a stimulus delivery tube, and the rat was placed in the cylindrical Plexiglas test chamber for a 5-min habituation period. Then a $1 \mathrm{ml}$ volume of the taste solution was infused into the mouth at a constant rate over $1 \mathrm{~min}$. Each rat was habituated to this procedure on two consecutive days prior to testing, using distilled water as the taste stimulus. The rats' behavior during the 1-min test trials was videotaped via a mirror mounted beneath the transparent floor of the test chamber.

\section{Behavioral Measures and Analysis}

The videotaped record for each rat was scored for the occurrence of positive (hedonic) and aversive actions. Strongly hedonic actions are paw licking, lateral (nonrhythmic) tongue protrusions, and rhythmic tongue protrusions along the midline. Neutral or compromise responses are mouth movements or passive drip of fluid from the mouth. Strongly aversive actions are gapes (large opening of the mandible and retractions of lower lip), chin rubbing (lowering mouth to floor and pushing forward), face washing (single wipe with the forepaws or a bout of several wipes), forelimb flails (shaking of the forelimb with a frequency greater than $60 \mathrm{~Hz}$ ), headshaking (at greater than $60 \mathrm{~Hz}$ ), paw treading (planting forelimbs on the floor and alternating forceful strokes forward and back), and rapid locomotion about the chamber [see $(3,19,20)$ for further details on the classification of these action patterns].

Videotapes were scored at $1 / 10$ speed by an observer blind to the drug condition of the rats. To quantify the number of responses emitted, discrete actions such as lateral tongue protrusions, gapes, chin rubs, and bouts of face washing, forelimb flailing, headshakes, paw treading, and locomotion were recorded each time they occurred. Continuous actions that persist for relatively long periods were recorded as follows: paw licks, mouth movements, and passive dripping were recorded in 5-sec bins (any occurrence of these behaviors up to 5-sec duration was counted as a single occurrence). Rhythmic tongue protrusions were scored in the same way in 2-sec bins. These data were analyzed with ANOVA followed by pair-wise comparisons of means.

\section{RESULTS}

\section{Positive Hedonic Reactions to Taste}

Hedonic reactions elicited by sucrose and by quinine were analyzed in a three-factor ANOVA (taste $\times$ drug $\times$ dose). As might be expected, sucrose and quinine tastes differed markedly in eliciting hedonic reactions, $\mathrm{F}(1,197)=69.57, p<0.0001$. Pharmacological agents proved to be a significant factor in modulating hedonic reactivity, $\mathrm{F}(6,197)=5.93, p<0.0001$, and there was a significant interaction between drug administration and taste quality, $\mathrm{F}(6,197)=4.57, p<0.001$. Two-factor ANOVAs (drug $\times$ dose) were performed separately for each taste in order to explore this interaction and to allow post hoc comparisons among the different pharmacological agents.

Sucrose in high, $\mathrm{F}(6,97)=7.52, p<0.0001$, and low concentrations, $\mathrm{F}(6,97)=5.46, p<0.001$, revealed a distinctive pattern 


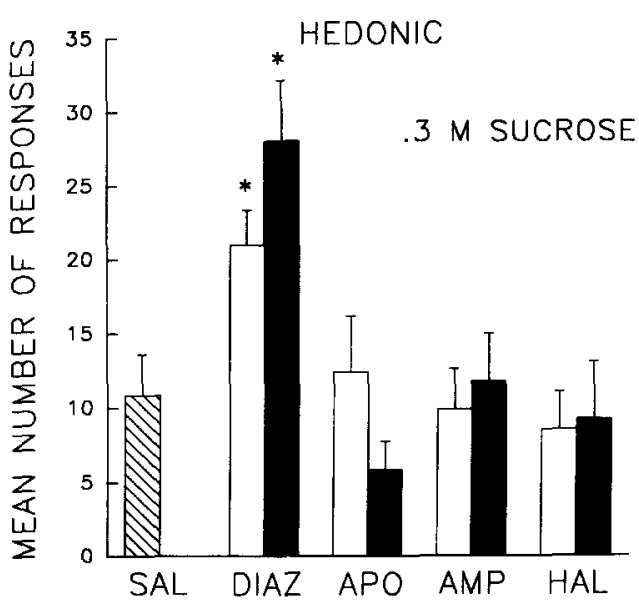

FIG. 1. Mean (SEM) number of hedonic reactions to sucrose $(0.3 \mathrm{M})$ after low (light bars) or high doses (dark bars) of diazepam (DIAZ), apomorphine (APO), amphetamine (AMP), haloperidol (HAL), or saline (SAL, striped bar).

of drug effects upon hedonic reactivity (Figs. 1 and 2). Diazepam increased hedonic reactivity to sucrose above saline baseline levels at both the 2.5 and $5.0 \mathrm{mg} / \mathrm{kg}$ doses $(p<0.05$ in each case, LSD tests). This hedonic potentiation of sucrose palatability by diazepam was the only reliable hedonic potentiation produced by any of the pharmacological agents. Dopaminergic agents failed to modulate sucrose hedonics either upwards or downwards: haloperidol, apomorphine, and amphetamine did not significantly alter sucrose hedonics in either direction at any dosage.

Because buspirone and gepirone were the last drugs administered for each rat, a possibility existed that order effects might contaminate any analysis which compared these drugs to saline control trials that were run early in the test series. In order to control for such order effects, a separate analysis was performed in which buspirone and gepirone trials were compared only to saline control trials that occurred within the last 5 trials for each rat

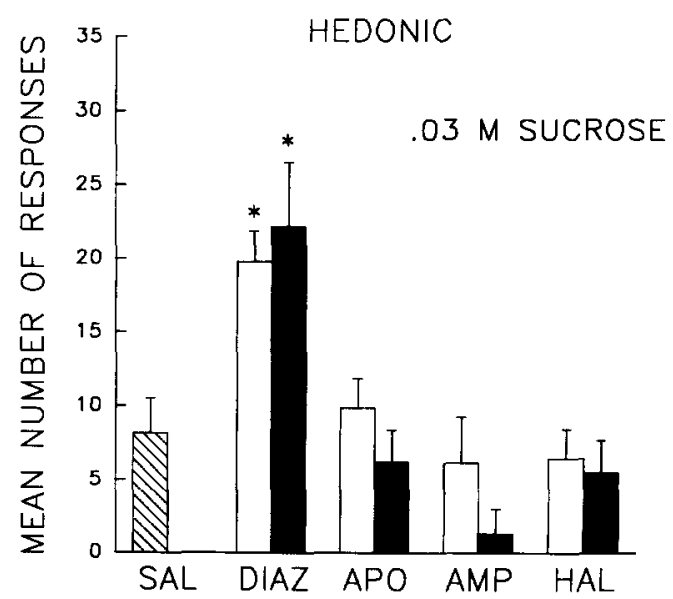

FIG. 2. Mean (SEM) number of hedonic reactions to sucrose $(0.03 \mathrm{M})$ after low (light bars) or high doses (dark bars) of diazepam (DIAZ), apomorphine, (APO), amphetamine (AMP), haloperidol (HAL), or saline (SAL, striped bar).

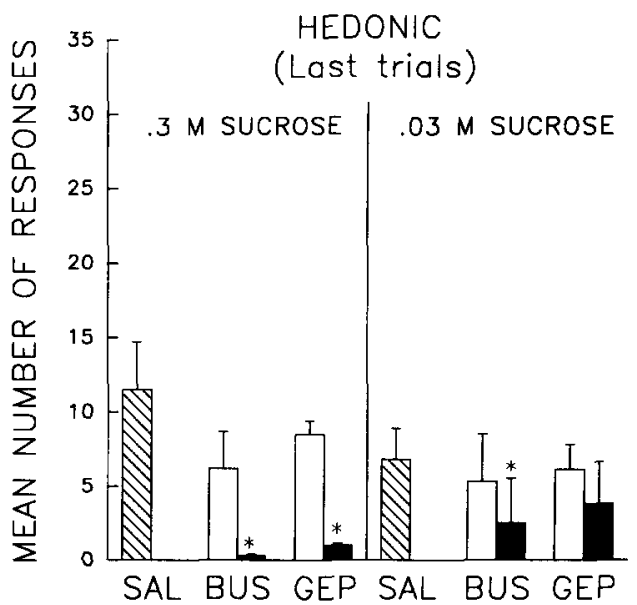

FIG. 3. Mean (SEM) number of hedonic reactions to $0.3 \mathrm{M}$ sucrose (left panel) or $0.03 \mathrm{M}$ sucrose (right panel) after low (light bars) or high doses (dark bars) of buspirone (BUS), gepirone (GEP) or saline (SAL, striped bar).

(Fig. 3). This analysis indicated that buspirone and gepirone exerted suppressive effects upon hedonic reactivity to sucrose, $\mathrm{F}(2,62)=3.96, p<0.05$

In contrast to the hedonic potentiation of sucrose palatability by diazepam, hedonic reactivity to bitter quinine remained unaffected by diazepam or by any other pharmacological agent, $F(6,97)=$ 1.8. This contrasts to the hedonic potentiation by chlordiazepoxide of a more dilute quinine solution $\left(3 \times 10^{-5} \mathrm{M}\right)$ found previously (4). The quinine solution used in this study was more concentrated by a factor of 10 , and was more unpalatable than the quinine solution used in the previous study. Overall hedonic reactions to quinine naturally were very low in this study compared to sucrose (mean $=1.0$ compared to sucrose mean $=10.8$ ), suggesting that hedonic potentiation by diazepam is less effective when initial hedonic levels are relatively low (i.e., hedonic potentiation by diazepam is multiplicative rather than additive).

\section{Aversive Reactions to Taste}

A three-factor ANOVA of aversive reactivity (taste $x$ drug $x$ dose) indicated that aversion too was elicited differently by sucrose and quinine, as expected, $\mathrm{F}(1,197)=48.32, p<0.0001$. Aversive reactivity to these tastes was modulated by pharmacological agents, $\mathrm{F}(6,197)=3.81, p<0.01$, and there was a significant interaction between taste and drug administration, $F(6,197)=$ $3.66, p<0.01$.

Separate two-factor ANOVAs (drug $\times$ dose) for each taste stimulus revealed that aversion was modulated pharmacologically only when initial levels of aversion exceeded very low levels. Highly concentrated sucrose, which elicited very low aversion overall (mean $=1.9$ aversive reactions), was not affected by drug administration, $F(6,97)=0.61$. Taste stimuli that elicited moderate to high levels of aversion overall, however, such as dilute sucrose [mean $=6.1$ aversive reactions; drug effect, $F(6,97)=$ $8.29, p<0.001$ ] and quinine [mean $=22.4$ aversive reactions; drug effect, $\mathrm{F}(6,97)=3.91, p<0.01]$, did show modulation of aversion by drug administration (Figs. 4 and 5). Post hoc tests showed that the diazepam-induced hedonic potentiation of dilute sucrose was accompanied by a reciprocal reduction in aversive reactivity $(p<0.05$, LSD test). Diazepam failed to reduce the higher aversive reactivity elicited by quinine, however, just as it failed to alter 


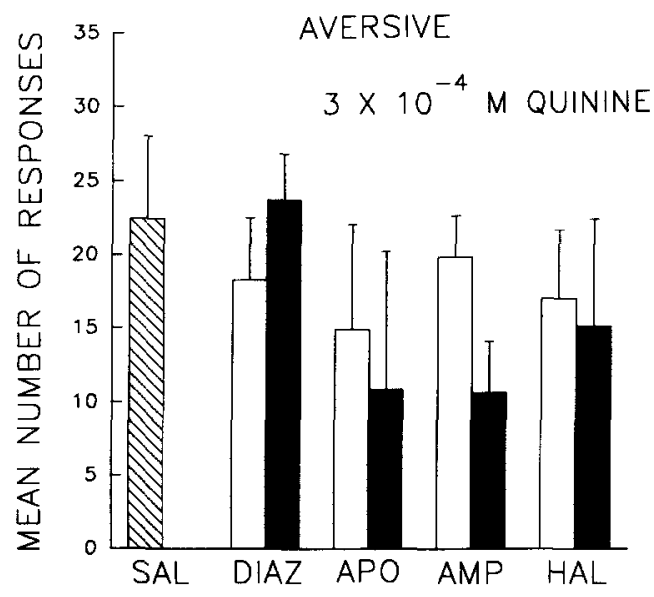

FIG. 4. Mean (SEM) number of aversive reactions to quinine after low (light bars) or high (dark bars) doses of diazepam (DIAZ), apomorphine (APO), amphetamine (AMP), haloperidol (HAL), or saline (SAL, striped bar).

hedonic reactions elicited by quinine. This supports our earlier suggestion that the effect of benzodiazepines, even for nonpreferred stimuli, is chiefly upon the hedonic, rather than the aversive limb of palatability. The reduction by diazepam of aversive responses emitted to dilute sucrose may simply be a secondary consequence of competition from the enhancement of hedonic reactions. The dopaminergic antagonist or agonists (haloperidol, apomorphine, amphetamine) did not alter aversive reactivity significantly for any taste, just as these agents failed to modulate hedonic reactivity.

The effects of buspirone and gepirone upon aversive reactivity were compared to saline control levels in a separate analysis, as was done for hedonic reactivity (Fig. 6). These serotonergic agents exerted a suppressive effect upon aversion, $F(2,62)=4.47$, $p<0.02$, which interacted with taste quality, $F(2,62)=4.61$, $p<0.02$. This effect and interaction mirrored those found for hedonic reactivity: the moderate to high levels of aversion elicited

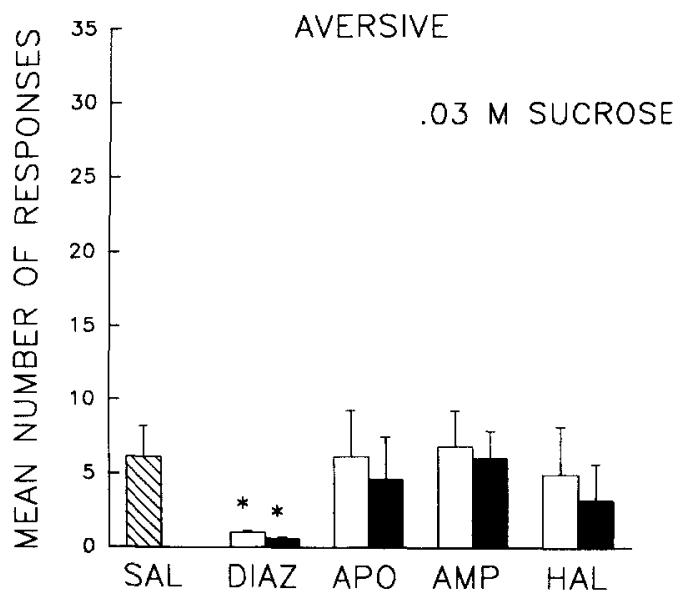

FIG. 5. Mean (SEM) number of aversive reactions to sucrose $(0.03 \mathrm{M}$ ) after low (light bars) or high (dark bars) doses of diazepam (DIAZ), apomorphine (APO), amphetamine (AMP), haloperidol (HAL), or saline (SAL, striped bar)

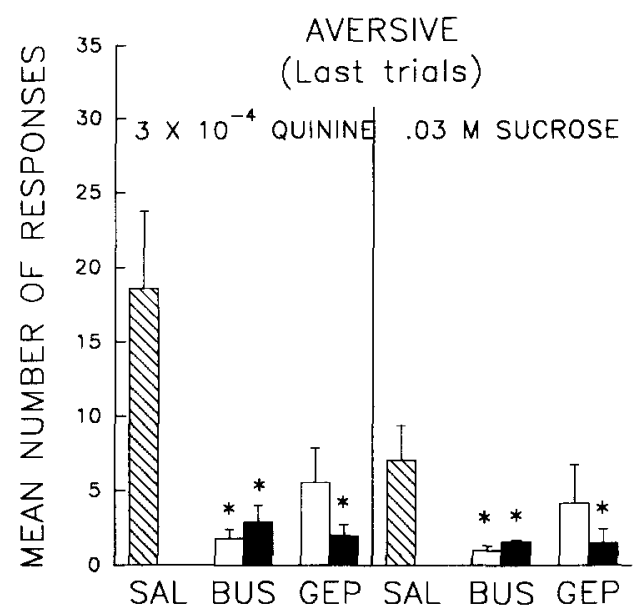

FIG. 6. Mean number (SEM) of aversive reactions to quinine (left panel) or $0.03 \mathrm{M}$ sucrose (right panel) after low (light bars) or high (dark bars) doses of buspirone (BUS), gepirone (GEP), or saline (SAL, striped bars).

by dilute sucrose or quinine were suppressed by buspirone and gepirone ( $p<0.05$ each, LSD tests), whereas the lower level of aversion elicited by highly concentrated sucrose was unaffected by these drugs.

\section{DISCUSSION}

The results of the present study suggest that a drug-induced enhancement of positive palatability may be a distinctive effect of benzodiazepine-type anxiolytics. Whereas diazepam amplified taste-elicited hedonic reactions, apomorphine, amphetamine, and haloperidol did not significantly change hedonic reactions in either direction, and buspirone and gepirone exerted only suppressive effects.

One of the most striking aspects of these results was the failure of dopaminergic agents to exert an effect upon either positive or aversive reactions. This failure stands in marked contrast to the well-documented capacity of these agents to control food intake and behavior on instrumental measures of reward. Disruption of dopaminergic transmission produced by either neuroleptic drugs or 6-hydroxydopamine brain lesions potently suppresses food intake $(28,34)$. Amphetamine, which stimulates dopaminergic systems, has been reported to stimulate feeding at low doses $(15,35)$, whereas both apomorphine and higher doses of amphetamine have been shown to suppress intake $(1,23)$. Furthermore, dopamine systems have been indicated to play a crucial role in mediating behavioral effects of rewards ranging from food to electrical brain stimulation: dopamine antagonists can suppress instrumental responding for many types of rewards $(16,37,38)$. Yet neither hedonic nor aversive reactions to tastes were changed reliably by these agents at any of the doses used in this study. The role of dopaminergic systems in appetite and reward appears to operate independently of basic taste affect, and may not involve a direct modulation of hedonic processes.

The implication that dopaminergic agents influence feeding and reward by acting upon a psychological process that is separable from basic affect is consistent with other evidence regarding dopamine systems and taste reactivity. The aphagia that follows the depletion of nigrostriatal dopamine projections by 6-OHDA, for example, can occur without a concomitant change in hedonic or aversive reactivity, suggesting that basic affect remains undiminished (6). Conversely, the elicitation of stimulation-bound feeding by electrical stimulation of the lateral hypothalamus, 
which is thought to involve dopamine projections $(22,26,27$, 29), occurs without an enhancement of taste hedonics (5). The stimulation or suppression of feeding by electrical brain stimulation, lesion, or pharmacological manipulation of brain dopamine systems, in other words, is accomplished via a psychological process that is separable from either food hedonics or aversion. This process may be a component of both natural appetite and reward, but is "downstream" from the basic affect generated by an encounter with rewarding or aversive stimuli (5).

Another surprising aspect of the present results was the failure of putative $5-\mathrm{HT}_{1 \mathrm{~A}}$ agonists to facilitate hedonic reactions: in contrast to diazepam, neither buspirone nor gepirone enhanced taste-elicited ingestive reactions. This is particularly noteworthy in view of the fact that buspirone, gepirone, and closely related $5-\mathrm{HT}_{1 \mathrm{~A}}$ agonists such as 8-OH-DPAT have been shown to enhance feeding in rats $(7,9,12-14,24)$. Thus, the drug-class specificity of the facilitation of hedonic taste reactivity apparently applies even within the class of clinical anxiolytics, separating benzodiazepine (e.g., diazepam) from nonbenzodiazepine anxiolytics (e.g., buspirone). The enhancement of positive palatability reactions by benzodiazepine agonists might be a unique drug effect not shared by compounds acting at other receptor sites.

It could be argued, however, that the failure to find significant enhancement of ingestive reactions by buspirone or gepirone was due to methodological limitations. For example, the dose, route of administration, or injection-test interval may not have been optimal to detect an enhancing effect of these agents on positive palatability. Be this as it may, the drug parameters used in the present study were not entirely dissimilar to those found effective in other studies [cf. $(7,24)]$. Another possibility is that the failure was due to an order effect, since buspirone and gepirone were tested last in an otherwise randomized series of drug trials. However, the separate analysis of the last 5 drug trials, which included these two agents, makes this interpretation unlikely.
Compared to taste reactivity seen under the saline control condition, buspirone and gepirone appeared to suppress taste reactivity in general. Whether or not this suppression is specifically due to an action at 5-HT $1 \mathrm{~A}$ receptor sites must await further studies which use more selective 5-HT ${ }_{1 \mathrm{~A}}$ agonists such as 8-OH-DPAT.

The special sensitivity of ingestive actions to diazepam supports our earlier suggestion that benzodiazepines promote feeding in part by selectively amplifying the perceived positive or hedonic palatability of foods rather than by suppressing the negative palatability of foods $(4,33)$. The observation that this effect of diazepam did not extend to $3 \times 10^{-4} \mathrm{M}$ quinine, a highly nonpreferred taste, suggests that this effect does not simply "add" hedonic palatability to a taste; instead, a minimum initial level of hedonic palatability appears to be required, which is then amplified by benzodiazepines.

In summary, drug-induced enhancement of positive palatability may be unique to benzodiazepine-type anxiolytics. Although more systematic study of the effects of $5-\mathrm{HT}_{1 \mathrm{~A}}$ agonists on taste reactivity is needed, at present the results with buspirone and gepirone do not suggest that this receptor system is strongly involved in the modulation of positive palatability. Similarly, DA agonists and antagonists do not appear to systematically affect palatability. Thus, the benzodiazepine receptor complex appears to play a special role in neural control of appetite through its modulation of the positive palatability of tastes [cf. ( 2 , $4,11,33)]$.

\section{ACKNOWLEDGEMENTS}

This work was supported by a grant from NIH awarded to K.C.B. and by grants from NSERC and PMHAC awarded to D.T. Gepirone and buspirone were donated by Bristol-Myers, Inc. The authors thank M. Mondloch for preparing the figures, and Dr. M. L. Spetch for her helpful comments on the manuscript.

\section{REFERENCES}

1. Barazahi, R.; Groppette, A.; Mantegazza, P.; Muller, E. E. Reduction of food intake by apomorphine: a pimozide-sensitive effect. J. Pharm. Pharmacol. 25:909-911; 1973.

2. Berridge, K. C. Brainstem mediates chlordiazepoxide enhancement of palatability. Brain Res. 447:262-268; 1988.

3. Berridge, K. C.; Grill, H. J. Isohedonic tastes support a two-dimensional hypothesis of palatability in rats. Appetite 5:221-231; 1984.

4. Berridge, K. C.; Treit, D. Chlordiazepoxide directly enhances positive ingestive reactions in the rat. Pharmacol. Biochem. Behav. 24: 217-221; 1986.

5. Berridge, K. C.; Valenstein, E. S. Hypothalamic stimulation that evokes feeding does not reliably amplify the hedonic evaluation of tastes. Soc. Neurosci. Abstr. 14:360; 1988.

6. Berridge, K. C.; Venier, I. L.; Robinson, T. E. Taste-reactivity analysis of 6-hydroxydopamine-induced aphagia: Implications for arousal and anhedonia hypothesis of dopamine function. Behav. Neurosci. 103:36-45; 1989 .

7. Clark, M.; Fletcher, A. Does buspirone elicit feeding by a similar mechanism to that of 8-OH-DPAT? Br. J. Pharmacol. 89:863P; 1986.

8. Cooper, S. J. Benzodiazepines as appetite-enhancing compounds. Appetite 1:7-19; 1980.

9. Cooper, S. J.; Desa, A. Benzodiazepines and putative 5-HT ${ }_{1 \mathrm{~A}}$ agonists increase hypertonic saline consumption in rehydrating rats. Pharmacol. Biochem. Behav. 28:187-191; 1987.

10. Cooper, S. J. Estall, L. B. Behavioral pharmacology of food, water and salt intake at benzodiazepine receptors. Neurosci. Biobehav. Rev. 9:5-19; 1985

11. Cooper, S. J.; Yerbury, R. E. Clonazepam selectively increases saccharin ingestion in a two-choice test. Brain Res. 456:173-176; 1988.

12. Dourish, C. T.; Hutson, P. H.; Kennett, G. A.; Curzon, G. 8-OHDPAT-induced hyperphagia: its neural basis and possible therapeutic relevance. Appetite 7(Suppl.):127-140; 1986.
13. Dourish, C. T.; Hutson, P. H.; Curzon, G. Putative anxiolytics 8OH-DPAT, buspirone and TVX Q 7821 are agonists at 5-HT 1A autoreceptors in the raphe nuclei. Trends Pharmacol. Sci. 7:212-214; 1986.

14. Dourish, C. T.; Hutson, P. H.; Curzon, G. Low doses of the putative serotonin agonist 8-hydroxy-2-(di-n-propylamino) tetralin (8-OHDPAT) elicit feeding in the rat. Psychopharmacology (Berlin) 86: 197-204: 1985 .

15. Evans, K. R.; Vaccario, F. J. Effects of d- and l-amphetamine on food intake: Evidence for a dopaminergic substrate. Pharmacol. Biochem. Behav. 27:649-652; 1987.

16. Fibiger, H. C.; Phillips, A. G. Dopamine and the neural mechanisms of reinforcement. In: Horn, A. S.; Korf, J.; Westerink, B. H., eds. The neurobiology of dopamine. London: Academic Press; 1979:597615.

17. Geary, N.; Smith, G. P. Pimozide decreases the positive reinforcing effect of sham-fed sucrose in the rat. Pharmacol. Biochem. Behav. 22:787-790; 1985 .

18. Gilbert, D. B.; Cooper, S. J. Effects of dopamine antagonists on fluid intake and salt preference in male and female rats. J. Psychopharmacol. 1:47-53; 1987.

19. Grill, H. J.; Berridge, K. C. Taste reactivity as a measure of the neural control of palatability. In: Sprague, J. M.; Epstein, A. N., eds. Progress in psychobiology and physiological psychology. Orlando, FL: Academic Press; 1985:1-61.

20. Grill, H. J.; Norgren, R. The taste-reactivity test: I. Mimetic response to gustatory stimuli in neurologically normal rats. Brain Res. 143:263-279; 1978

21. Hommer, D. W.; Skolnick, P.; Paul, S. M. The benzodiazepine/gaba receptor complex and anxiety. In: Meltzer, H. Y., ed. Psychopharmacology: The third generation of progress. New York: Raven Press; 1987:977-983. 
22. Mittleman, G.; Castaneda, E.; Robinson, T. E.; Valenstein, E. S. The propensity for non-regulatory ingestive behavior is related to differences in dopamine systems: behavioral and biochemical evidence. Behav. Neurosci. 100:213-220; 1986.

23. Muscat, R.; Willner, P.; Towell, A. Apomorphine anorexia: a further pharmacological characterization. Eur. J. Pharmacol. 123:131; 1986.

24. Neill, J. C.; Cooper, S. J. MDL 72832, a selective 5-HT 1A receptor ligand, stereospecifically increases food intake. Eur. J. Pharmacol. 151:329-332; 1988.

25. Pelchat, M. L.; Grill, H. L.; Rozin, P.; Jacobs, J. Quality of acquired responses to tastes by Rattus norvegicus depends on type of associated discomfort. J. Comp. Psychol. 97:140-153; 1983.

26. Phillips, A. G.; Fibiger, H. C. Dopaminergic and noradrenergic substrates of positive reinforcement: differential effects of d- and l-amphetamine. Science 179:575-577; 1973.

27. Phillips, A. G.; Nikaido, R. S. Disruption of brain stimulation-induced feeding by dopamine receptor blockade. Nature 258:750-751; 1975.

28. Rolls, E. P.; Rolls, B. J.; Kelly, P. H.; Shaw, S. G.; Wood, R. J.; Dale, $R$. The relative attenuation of self-stimulation, eating and drinking produced by dopamine receptor blockade. Psychopharmacology (Berlin) 38:219-230; 1974.

29. Rowland, N.; Marques, D. M.; Fisher, A. E. Comparison of the effects of brain dopamine-depleting lesions upon oral behaviors elicited by tail pinch and electrical brain stimulation. Physiol. Behav. 24: $273-281 ; 1980$.

30. Rusk, I. N.; Cooper, S. J. The selective dopamine DI receptor ago- nist SK\&F 38393: Its effects on palatability- and deprivation-induced feeding, and operant responding for food. Pharmacol. Biochem. Behav. 34:17-22; 1989.

31. Tombaugh, T. N.; Tombaugh, J.; Anisman, H. Effects of dopamine receptor blockade on alimentary behaviors: home cage food consumption, magazine training, operant acquisition, and performance. Psychopharmacology (Berlin) 66:219-225; 1979.

32. Treit, D. Animal models for the study of anti-anxiety agents: A review. Neurosci Biobehav. Rev. 9:203-222; 1985.

33. Treit, D.; Berridge, K. C.; Schultz, C. E. The direct enhancement of positive palatability by chlordiazepoxide is antagonized by Ro 151788 and CGS 8216. Pharmacol. Biochem. Behav. 26:709-714; 1987.

34. Ungerstedt, U. Adipsia and aphagia after 6-hydroxydopamine induced degeneration of the nigro-striatal dopamine system in the rat brain. Acta Physiol. Scand. 82(Suppl. 367):95-122; 1971.

35. Winn, P.; Williams, S. F.; Herberg, L. J. Feeding stimulated by very low doses of d-amphetamine administered systemically or by microinjection into the striatum. Psychopharmacology (Berlin) 78:336-341; 1982.

36. Wise, R. A. The anhedonia hypothesis: Mark III. Behav. Brain Sci. 8:178-186; 1985.

37. Wise, R. A.; Spindler, J.; de Wit, H.; Gerber, G. J. Neuroleptic-induced 'anhedonia' in rats: Pimozide blocks the reward quality of food. Science 210:262-264; 1978.

38. Xenakis, S.; Sclafani, A. The effects of pimozide on consumption of a palatable saccharin-glucose solution in the rat. Pharmacol. Biochem. Behav. 15:435-442; 1981. 